\title{
Intrinsic anomalous surface roughening of TiN films deposited by reactive sputtering
}

\author{
M. A. Auger, ${ }^{1,5}$ L. Vázquez, ${ }^{1, *}$ R. Cuerno, ${ }^{2}$ M. Castro, ${ }^{3}$ M. Jergel,${ }^{4}$ and O. Sánchez ${ }^{1}$ \\ ${ }^{1}$ Instituto de Ciencia de Materiales de Madrid-CSIC, C Sor Juana Inés de la Cruz 3, E-28049 Cantoblanco, Madrid, Spain \\ ${ }^{2}$ Departamento de Matemáticas y Grupo Interdisciplinar de Sistemas Complejos, Universidad Carlos III de Madrid, \\ Avenida de la Universidad 30, E-28911, Leganés, Madrid, Spain \\ ${ }^{3}$ Grupo Interdisciplinar de Sistemas Complejos y Grupo de Dinámica No Lineal, Escuela Técnica Superior de Ingeniería (ICAI), \\ Universidad Pontificia Comillas de Madrid, E-28015, Madrid, Spain \\ ${ }^{4}$ Institute of Physics, Slovak Academy of Sciences, Dubravska cesta 9, 84511 Bratislava 45, Slovak Republic \\ ${ }^{5}$ Centro Nacional de Investigaciones Metalúrgicas (CENIM-CSIC), Avda. Gregorio del Amo, 8, E-28040 Madrid, Spain
}

\begin{abstract}
We study surface kinetic roughening of TiN films grown on $\mathrm{Si}(100)$ substrates by dc reactive sputtering. The surface morphology of films deposited for different growth times under the same experimental conditions were analyzed by atomic force microscopy. The TiN films exhibit intrinsic anomalous scaling and multiscaling. The film kinetic roughening is characterized by a set of local exponent values $\alpha_{\mathrm{loc}}=1.0$ and $\beta_{\mathrm{loc}}=0.39$, and global exponent values $\alpha=1.7$ and $\beta=0.67$, with a coarsening exponent of $1 / z=0.39$. These properties are correlated to the local height-difference distribution function obeying power-law statistics. We associate this intrinsic anomalous scaling with the instability due to nonlocal shadowing effects that take place during thin-film growth by sputtering.
\end{abstract}

DOI: 10.1103/PhysRevB.73.045436

PACS number(s): 68.55.- a, 81.15.Cd, 81.15.Aa, 68.35.Ct

\section{INTRODUCTION}

Kinetic interface roughening under nonequilibrium conditions has been an issue of considerable interest in the last several years. ${ }^{1,2}$ In particular, efforts have focused on the study of surface roughening for both growth and erosion (or etching) of thin films, ${ }^{2-4}$ which usually take place under farfrom-equilibrium conditions. This interest is explained by the relevance of surface film characterization at the submicron level, as well as to the search of the mechanisms that determine the film morphology and can contribute to achieving better control of the film properties. Despite the complexity and diversity of these phenomena, theoretical and experimental studies have indeed shown the existence of kinetic surface roughening in many of these systems. ${ }^{1,3}$ In addition, several studies have revealed the occurrence of scaling exponents that, in many cases, correspond to a few universality classes. However, nowadays, the general picture of kinetic roughening in growth and erosion systems is far from being fully understood. ${ }^{4}$ In spite of the fact that many systems do show kinetic roughening, their exponents are not explained by any of the existing theoretical models. At this stage of the research, for such systems a detailed analysis of the interface dynamics is required in order to be able to fully characterize them, the determination of the most complete set of experimental data being critical in order to better compare them to models and simulations.

With this aim we have studied the growth dynamics of titanium nitride films (TiN) deposited by reactive sputtering. The interest of this material lays in its wide range of applications, such as protective material for industrial tools, ${ }^{5}$ diffusion barriers in semiconductor technology, ${ }^{6}$ a gate electrode in transistors, ${ }^{7}$ a contact layer in solar cells, ${ }^{8}$ etc. This high performance of $\mathrm{TiN}$ is due to its excellent physical properties: high hardness, excellent tribological behavior, thermal and chemical stability, etc., which in some cases can be related to the film morphology. For instance, in the use of TiN films as diffusion barriers, the response of the film strongly depends on its microstructure ${ }^{9}$ because it can affect the oxygen incorporation in the layer due to density variations or the presence of porosity. Among the different deposition techniques usually applied to grow TiN films, the most widely used one is reactive sputtering, which has been used in the present work.

\section{Scaling concepts}

Let us consider a generic surface being described by a height field, $h(\vec{r}, t)$. The theory of dynamic scaling allows us to characterize the surface by determining the spatial and temporal correlations of the global interface width, $W(L, t)$, computed over a region of size $L, W(L, t)$ $=\left\langle[h(\vec{r}, t)-\langle h(\vec{r}, t)\rangle]^{2}\right\rangle^{1 / 2}$ in which the angular brackets denotes spatial averaging. Alternatively, the system dynamics can be also characterized by the so-called local interface width, $w(l, t), l$ being the size of an observation window, $l$ $\ll L$. The standard Family-Vicsek (FV) dynamic scaling is represented by the scaling ansatz ${ }^{1}$

$$
w(l, t)=t^{\alpha / z} f\left(l / t^{1 / z}\right)
$$

where the scaling function $f(u)$ behaves as:

$$
f(u) \propto\left\{\begin{array}{cl}
u^{\alpha} & \text { if } u \ll 1 \\
\text { const } & \text { if } u \gg 1
\end{array} .\right.
$$

Thus, for $l \ll t^{1 / z}, w$ scales as $l^{\alpha}$ while, for $l \gg t^{1 / z}$, it scales as $t^{\beta}(\alpha / z=\beta)$. The crossover between these behaviors takes place when the lateral correlation length $\xi$, which indicates the extent of the lateral correlations of the system, is comparable to the observation window. $\xi$ scales with time as $t^{1 / z}$. The FV scaling and the assumption of the self-affinity of the 
surface imply that the local interface width $w(l, t)$ scales as the global width.

This scaling framework has been successfully applied to several experimental systems..$^{1,2,10-12}$ However, it has become evident, both theoretically and experimentally, that it is not always valid. ${ }^{4,13}$ In fact, it has been found that for some systems the local and global widths scale differently. ${ }^{13-20}$ Thus, whereas the global width scaling is given by Eqs. (1) and (2), the local width obeys the following scaling relation:

$$
w(l, t)=t^{\alpha / z} f_{A}\left(l / t^{1 / z}\right),
$$

where the scaling function $f_{A}(u)$ behaves as:

$$
f_{A}(u) \propto\left\{\begin{array}{ll}
u^{\alpha_{\text {loc }}} & \text { if } u \ll 1 \\
\text { const } & \text { if } u \gg 1
\end{array} .\right.
$$

This scaling behavior, which is known as anomalous scaling, is characterized by a set of independent, local, roughness, and growth exponents, $\alpha_{\mathrm{loc}}$ and $\beta_{\mathrm{loc}}$, respectively, with $\alpha_{\mathrm{loc}} / z=\beta_{\mathrm{loc}}$, which characterize the local surface fluctuations on the scales $r \ll L$ and a set of global ones, $\alpha$ and $\beta$, with $\alpha / z=\beta$. It should be reminded that, for the FV scaling case, $\alpha_{\mathrm{loc}}=\alpha$ and $\beta_{\mathrm{loc}}=\beta$.

The scaling behavior can be determined by studying the structure factor or power spectral density (PSD) of the interfacial fluctuations, defined as $\operatorname{PSD}(k, t)=\langle H(k, t) H(-k, t)\rangle$, where $H(k, t)$ is the Fourier transform of the surface height in a system of lateral size $L$, with $k$ being the spatial frequency in reciprocal space. It can be shown ${ }^{13}$ that, for an anomalous 2+1-dimensional system, the PSD function follows the scaling relation:

$$
\operatorname{PSD}(k, t)=k^{-(2 \alpha+2)} f_{P}\left(k t^{1 / z}\right)
$$

where

$$
f_{P}(p) \propto\left\{\begin{array}{cc}
p^{2 \alpha+2} & \text { if } p \ll 1 \\
p^{2\left(\alpha-\alpha_{S}\right)} & \text { if } p \gg 1
\end{array},\right.
$$

$\alpha_{s}$ being known as the spectral roughness exponent. ${ }^{14}$ From the different sets of $\alpha, \alpha_{\text {loc }}$, and $\alpha_{s}$ values, up to four scaling behaviors are expected. ${ }^{14}$ Three of them have already been observed experimentally, ${ }^{4}$ namely, (i) FV standard scaling, which occurs when $\alpha=\alpha_{\mathrm{loc}}=\alpha_{s}<1$; (ii) intrinsic anomalous scaling, which takes place when $\alpha \neq \alpha_{\mathrm{loc}}=\alpha_{s} \leqslant 1$; and (iii) superrough scaling for $1=\alpha_{\mathrm{loc}} \neq \alpha=\alpha_{s}>1$.

In this work we will focus our attention on the second case, which seems to be applicable to our system. By introducing the values for the exponents in Eqs. (5) and (6), we can write

$$
\operatorname{PSD}(k, t) \propto\left\{\begin{array}{cc}
t^{(2 \alpha+d) / z} & p \ll 1 \\
k^{-\left(2 \alpha_{\mathrm{loc}}+d\right)} t^{2\left(\alpha-\alpha_{\mathrm{loc}}\right) / z} & p \gg 1
\end{array},\right.
$$

where $d=2$ is the dimension of the substrate. This behavior implies that $\operatorname{PSD}(k, t)$ curves obtained for different times do not overlap for large $k$ values, since $\alpha-\alpha_{\text {loc }} \neq 0$, i.e., the local and global widths scale differently. Since $\left(\alpha-\alpha_{\text {loc }}\right) / z$ $=\beta-\beta_{\mathrm{loc}}=\beta^{*}, \beta^{*}$ is known as the local anomalous growth exponent, describing the degree of anomaly of the system.
Thus, anomalous scaling in a system implies that $\operatorname{PSD}(k, t)$ curves should shift vertically upward as the growth time increases. It can be shown that a similar behavior should also be observed for the height-height correlation function, $G_{2}(r, t)$, curves, this function being defined as

$$
G_{2}(r, t)=\left\{\left\langle\left[h\left(\vec{r}^{\prime}+\vec{\ell}, t\right)-h\left(\vec{r}^{\prime}, t\right)\right]^{2}\right\rangle\right\}^{1 / 2} .
$$

In this work, we address the study of the scaling behavior of TiN films grown by dc reactive sputtering under this generalized theoretical framework. The surface morphology of the films deposited for different times has been analyzed by atomic force microscopy (AFM). The different functions mentioned above have been measured and studied in order to properly identify the growth mode.

\section{EXPERIMENTAL}

TiN films were deposited on (100) oriented silicon substrates at low temperature $(<70 \mathrm{C})$ in a reactive dc magnetron sputtering system described previously. ${ }^{21}$ The targetsubstrate distance was $6.5 \mathrm{~cm}$. Sputtering from a high-purity $(99.995 \%)$ titanium target was performed at $80 \mathrm{~W}$ dc discharge power in an $\mathrm{Ar}$ and $\mathrm{N}_{2}$ working gas mixture. The base pressure was lower than $10^{-6} \mathrm{mbar}$, and the working pressure was $\sim 2 \times 10^{-3}$ mbar when using the selected gas mixture of $11 \mathrm{sccm}$ (standard cubic centimeter per minute) total flow $\left(93 \% \mathrm{Ar}, 7 \% \mathrm{~N}_{2}\right.$ ). These deposition conditions enable us to grow stoichiometric TiN films $\left(\mathrm{TiN}_{x}\right.$ with $x$ close to 1$)$ at a constant growth rate of $5 \mathrm{~nm} / \mathrm{min}$. In order to study the roughness evolution of TiN, a series of films was grown at deposition times ranging from $24 \mathrm{~min}$ up to $200 \mathrm{~min}$.

The samples were imaged in air by atomic force microscopy (AFM) using a Nanoscope IIIa (Digital Instruments, $\mathrm{CA}$ ) equipment operating in the tapping mode. Silicon cantilevers with a nominal radius of curvature of $10 \mathrm{~nm}$ were employed. Note that, due to the relative hardness of the TiN films $(\sim 20 \mathrm{GPa})$, the tapping-mode AFM imaging is more reliable than contact-mode imaging since the tip-sample interaction is considerably lower, leading to less tip damaging and, therefore, to less tip convolution effects. AFM images with a resolution of $512 \times 512$ pixels were used for the calculations shown below, over scanning areas of 500 $\times 500 \mathrm{~nm}^{2}$, which are well above the largest observed correlation length to be statistically relevant.

X-ray-diffraction analysis of the films revealed that the preferred orientation of the films is (311) during the whole growth process.

\section{RESULTS}

Figure 1 shows three typical AFM images of TiN films for different deposition times. In addition, Figure 1(d) shows the normalized height distribution $P^{*}(h)\left(P^{*}(h)\right.$ $\left.\equiv P(h) / \int P(h) d h\right)$ of the AFM images corresponding to the thickest TiN film. Inspection of these data shows that the surface is not up-down symmetric, as indicated by the asymmetric shape with pronounced negative tail of $P^{*}(h)$, which leads to a negative value of the surface height skewness $S$, 

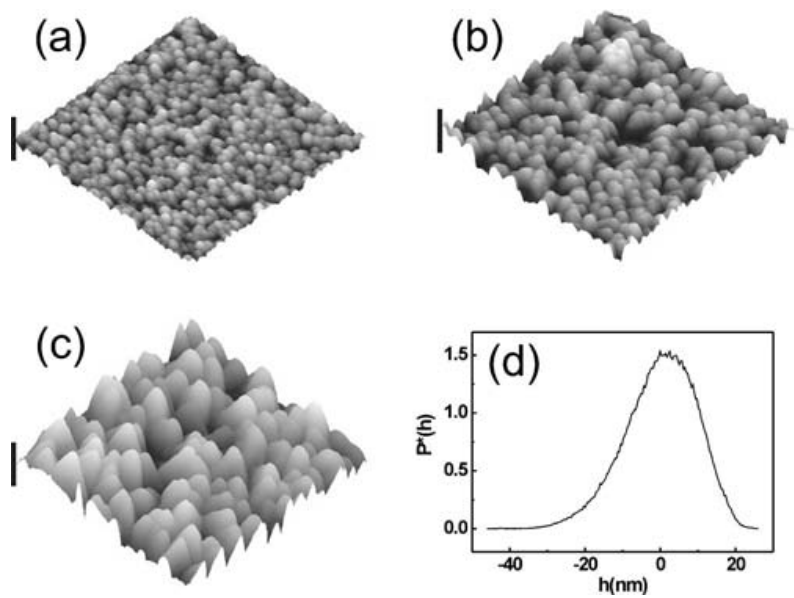

FIG. 1. Tapping mode $500 \times 500 \mathrm{~nm}^{2}$ AFM images of TiN films grown, under the same experimental conditions for: (a) 24 min., (b) $72 \mathrm{~min}$., (c) $200 \mathrm{~min}$. The vertical bar represents $50 \mathrm{~nm}$. (d) Normalized height distribution, $P^{*}(h)$, obtained from the AFM data corresponding to the thickest TiN film.

$S=\int h^{3} P^{*}(h) d h / W^{3}$. This asymmetry should reflect into the continuum modeling of the system by the existence of (nonlinear) terms that are not symmetric under height reflection $h \leftrightarrow-h .^{22}$ In our experiments, $P^{*}(h)$ becomes more asymmetric as growth proceeds (not shown), which is consistent with the fact that these terms dominate the growth evolution for longer growth times.

From the AFM images, both the surface roughening and the coarsening process of the observed granular structure are evident. The root-mean-square roughness (i.e., the global surface width) and the average grain size (i.e., typical lateral correlation length) can be obtained from the AFM data. These data (error bars reflect statistics) are plotted in Fig. 2 as functions of deposition time, from which we can obtain the values of the growth exponent, $\beta=0.67 \pm 0.04$ and the coarsening exponent, $1 / z=0.39 \pm 0.02$. In order to asses the scaling behavior of our system, we have analyzed the $\operatorname{PSD}(k, t)$ functions corresponding to the films deposited for different times, as shown in Fig. 3. All the PSD curves have a similar shape. They present a linear dependence (in the logarithmic plot) for large $k$ values, which indicates the ex-

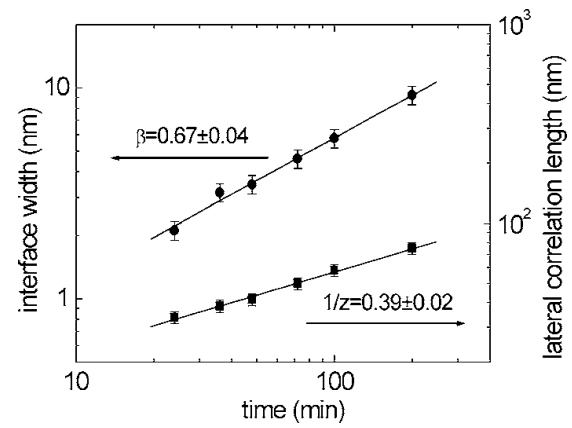

FIG. 2. Logarithmic plots of the interface width (top data, left $y$ axis) and the lateral correlation length (bottom data, right $y$ axis) versus deposition time. The values of $\beta$ and $1 / z$ are indicated.

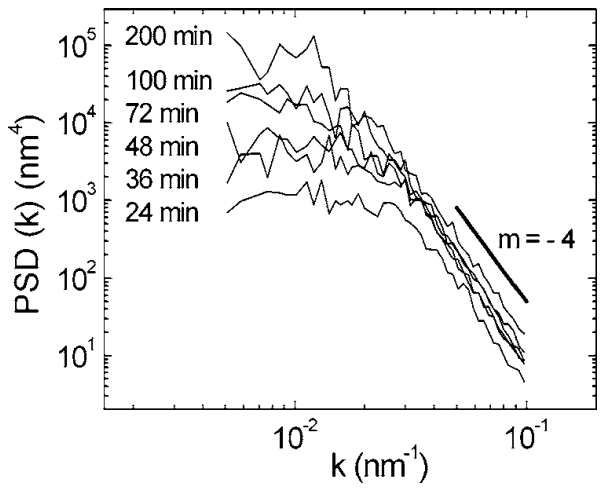

FIG. 3. Logarithmic plot of $\operatorname{PSD}(k, t)$ functions versus wave number $k$, for different growth times. The slopes of the PSD curves for large $k$ values are indicated. The deposition time for each curve is provided.

istence of correlations of the surface roughness for these short length scales $(l=1 / k)$. In contrast, for small $k$ values, they run (with some fluctuations) parallel to the $\mathrm{x}$ axis, (i.e., at the corresponding times the surface roughness does not show any spatial correlation for these larger length scales). The crossover between both regions shifts to smaller $k$ values (i.e., to larger spatial scales) as deposition time increases, in agreement with the coarsening process of the lateral correlation length found in Fig. 2. Clearly, the PSD functions are shifted upward as deposition time increases, which is a clear indication that the system presents intrinsic anomalous scaling behavior. ${ }^{13}$ In fact, the $G_{2}(r, t)$ functions display a similar behavior (not shown), which is consistent with this scaling behavior. ${ }^{13}$ From the slope of PSD curves for large $k$ values and within statistical errors, we obtain a value of the local roughness exponent, $\alpha_{\mathrm{loc}}$ close to $1, \alpha_{\mathrm{loc}}=1.0 \pm 0.1$. The value of $\alpha$ (global) can be obtained from the relationship $1 / z$ $=\beta / \alpha$. This calculation leads to $\alpha=1.7 \pm 0.1$. Also, from the values of $\alpha_{\text {loc }}$ and $1 / z$, we obtain $\beta_{\text {loc }}=0.39 \pm 0.05$ and, therefore, $\beta^{*} \approx 0.28$.

In order to further verify that the scaling behavior of our system corresponds to intrinsic anomalous scaling, both qualitatively and quantitatively, we can collapse the PSD functions obtained for the various deposition times. Namely, from Eq. (7) it follows that, plotting the curves $\operatorname{PSD}(k, t) k^{(2 \alpha+d)}$ versus $k t^{1 / z}$ for all growth times, all the curves must collapse into a single one. Specifically, the slope of this single curve equals $m_{1}=2 \alpha+2$ for small $k t^{1 / z}$ values, and $m_{2}=2\left(\alpha-\alpha_{\text {loc }}\right)$ for large $k t^{1 / z}$ values. Thus, the collapse of these functions is a verification of the consistency of the analysis. ${ }^{13}$ The collapse of the PSD curves is displayed in Fig. 4. It is clear that, within the experimental error, all the curves indeed collapse into a single curve with two slopes. The slope for small abscissas is $m_{1}=5.6 \pm 0.1$, whereas for large abscissas it takes the value $m_{2}=1.40 \pm 0.05$. It is worth noting that $m_{2} \neq 0$ already implies $\alpha_{\text {loc }} \neq \alpha$ and, therefore, anomalous scaling is present. Furthermore, we can obtain the values of $\alpha_{\text {loc }}$ and $\alpha$ from the above expressions for $m_{1}$ and $m_{2}$. This results into $\alpha_{\text {loc }}=1.1 \pm 0.1$ and $\alpha=1.80 \pm 0.05$, which are consistent, within the error bars, with the values obtained above. 


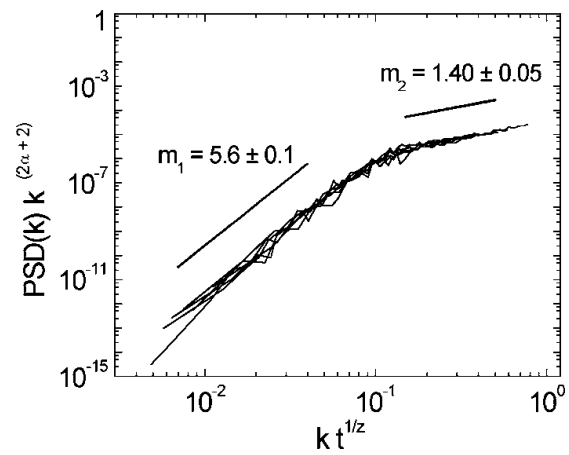

FIG. 4. Logarithmic plot of $\operatorname{PSD}(k, t) k^{(2 \alpha+2)}$ vs $k t^{1 / z}$ for different growth times showing the good collapse of the $\operatorname{PSD}(k, t)$ curves. The straight lines indicate the values of the slopes of the collapsed curves for small and large $k t^{1 / z}$ arguments.

One open issue connected with anomalous scaling systems is the relation to multiscaling properties. Thus, below we check if our system presents also multiscaling by studying the scaling of the higher $(q$ th)-order height difference correlation functions, which are defined as

$$
G_{q}(r, t)=\left\{\left\langle[h(\vec{r}+\vec{\ell}, t)-h(\vec{r}, t)]^{q}\right\rangle\right\}^{1 / q} \sim r^{\alpha} q,
$$

$\alpha_{q}$ being generalized local roughness exponents. We want to remark that, in the case of FV scaling, $\alpha_{q}=\alpha \forall q \geqslant 2$. In contrast, when the system exhibits multiscaling, the value of $\alpha_{q}$ changes with $q$. The results obtained in our system for $G_{q}(r, t)$ are shown in Fig. 5 for $q=2,3, \ldots, 8$. We obtain a progressive decrease of the measured exponent, varying from $\alpha_{2}=0.91$ to $\alpha_{8}=0.57$. This behavior, which has been also found, e.g., in imbibition experiments, ${ }^{12,20}$ confirms the existence of both multiscaling and anomalous scaling in our system. The fact that $\alpha_{2}=0.91<1$ is likely explained by computational limitations of the $G_{2}(r, t)$ function, which makes it hard to obtain values of $\alpha_{\text {loc }}$ close to $1 .^{23-25}$ In order to reliably check this limitation, we have performed a numerical integration of the linear molecular beam epitaxy (MBE) growth equation (for a two-dimensional substrate),

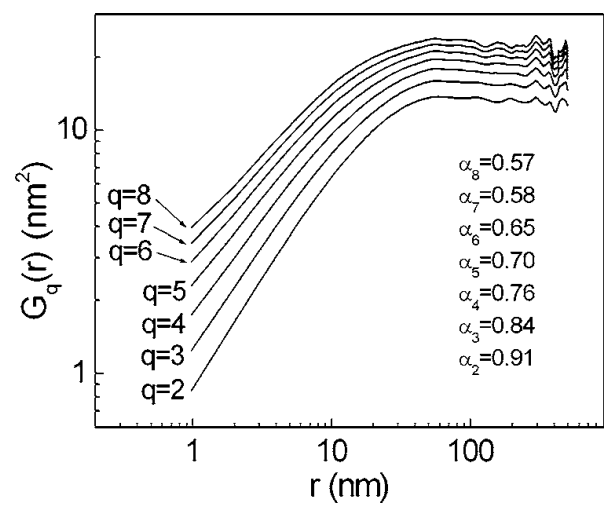

FIG. 5. Multiscaling analysis through the computation of the $q$-order height difference correlation function for the TiN thickest film surface. The corresponding $\alpha_{q}$ values are indicated.

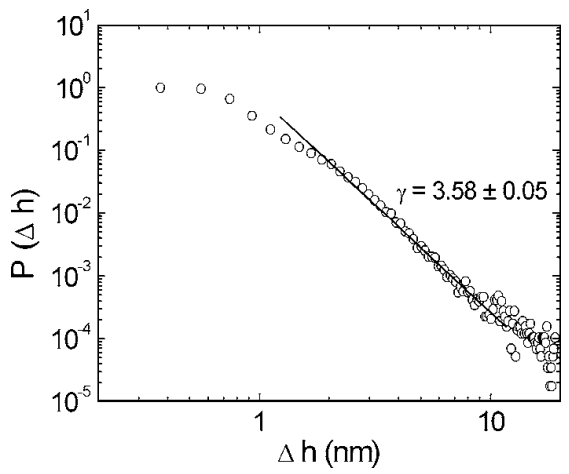

FIG. 6. Logarithmic plot of $P(\Delta h)$ vs $\Delta h$ obtained from the AFM images of the thickest TiN film. The straight line indicates the region in which $P(\Delta h) \propto(\Delta h)^{-\gamma}$, as well as the value of $\gamma$.

which can be solved analytically and is known to follow a super-rough behavior ${ }^{13,15,26}$ with $\alpha_{\text {loc }}=1$. When we evaluated the corresponding $G_{2}(r, t)$ function, we also obtained a value clearly smaller than 1 , whereas we obtained the expected values for the other exponents.

Recently, it has been suggested that the morphological properties of the slope distribution function, $P(\Delta h)$, where $\Delta h=\left\langle\left|h\left(r_{i+1}\right)-h\left(r_{i}\right)\right|\right\rangle$, is closely related to intrinsic anomalous scaling as long as $P(\Delta h)$ follows a power-law behavior. ${ }^{18,19}$ This kind of behavior is due to the relatively large abundance of large jumps in the interface values. Specifically, for one-dimensional systems in which $P(\Delta h)$ $\sim(\Delta h)^{-\gamma}$ with $0<\gamma<3$, it has been shown that the interface will present both anomalous scaling and multiscaling. ${ }^{18,19}$ Accordingly, we have calculated $P(\Delta h)$ for the thickest TiN film. In Fig. 6, we have plotted in a logarithmic scale $P(\Delta h)$ versus $\Delta h$ in order to verify whether for our system $P(\Delta h)$ follows a power-law behavior. Clearly, there is a linear region (in the logarithmic plot), spanning from $\Delta h=2$ to $\Delta h$ $=10$, in which $\gamma=3.58 \pm 0.05$. Moreover, the analysis of $P(\Delta h)$ allows us to further check the reliability of our scaling analysis. This can be done through the study of the scaling of the mean local slopes with time, $\rho(t)=\left[\left\langle(\Delta h)^{2}\right\rangle\right]^{1 / 2}{ }^{20}$ In the presence of anomalous scaling, $\rho(t)$ scales with time as $\rho(t) \sim t^{\beta^{*}} .{ }^{13}$ In Fig. 7, we plot $\rho(t)$, as computed from $P(\Delta h)$, versus time in a log-log scale. The slope gives $\beta^{*}$ $=0.25 \pm 0.04$, which agrees, within the error bars, with the value obtained previously. Beyond confirming the validity and consistency of our scaling analysis, these results show that for $2+1$ surface film growth systems, the existence of intrinsic anomalous scaling can appear associated with a local surface slope distribution following a power-law behavior, thus, confirming the fact that anomalous interfaces present, statistically, larger jumps in height than interfaces with FV scaling.

\section{DISCUSSION AND CONCLUSIONS}

From the previous analysis, we can draw the following conclusions concerning the growth dynamics of TiN films deposited by reactive sputtering. First, this system exhibits 


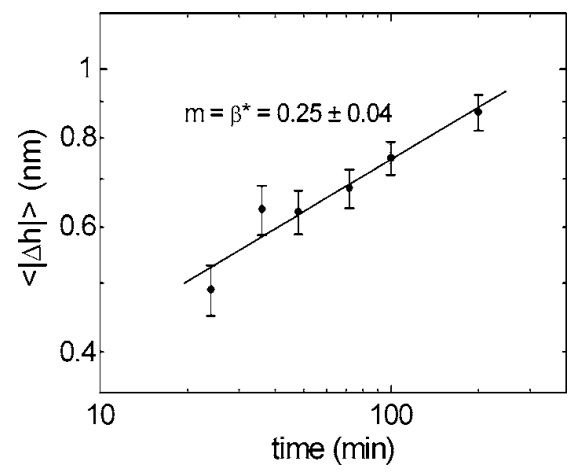

FIG. 7. Mean local slope as a function of growth time, as computed from Fig. 6 . The corresponding $\beta^{*}$ value is indicated.

intrinsic anomalous scaling characterized by a coarsening exponent value $1 / z=0.39$, related to the average grain size observed in the AFM images. The local width is characterized by $\alpha_{\mathrm{loc}}=1.0$ and $\beta_{\mathrm{loc}}=0.39$, whereas the global width shows the scaling exponents $\alpha=1.7$ and $\beta=0.67$, which implies a local anomalous growth exponent value of $\beta^{*} \approx 0.28$. Second, the system displays multiscaling, which is usually related to anomalous scaling. ${ }^{27}$ Finally, these properties of the film surface morphology appear to be due to the relatively large jumps in the interface position, characterized by a local slope distribution displaying a power-law behavior.

One of the goals of the dynamic scaling theory of rough interfaces lays on its capability to infer the underlying physical mechanisms involved from the value of the roughening exponents. Hence, it is interesting to try to gather further insight about the physical origin of the intrinsic anomalous scaling found for our experimental system. In general, this issue is not well understood for experimental systems in the field of surface growth. Thus, anomalous scaling has recently been observed during electrodeposition of $\mathrm{Cu}^{17}$ and $\mathrm{Ni}^{28}$ and $\mathrm{Cu}$ electroless deposition. ${ }^{29}$ Former studies suggest that anomalous scaling was also present for the physical and chemical vapor deposition of metals $\mathrm{s}^{30,31}$ and polymer films prepared by vapor deposition. ${ }^{32}$ Finally, superrough anomalous behavior was described for Pt sputtered films under simultaneous substrate rotation. ${ }^{16}$

Although there is no well-established theory of anomalous scaling, some recent studies suggest that it must be due to nonlocal effects in the surface dynamics. ${ }^{33}$ Notwithstanding, it is not clear whether such nonlocal effects are responsible for the destabilization of the surface or for the origin of anomalous scaling. Besides, the nonlocality can be induced by different effects such as, for instance, diffusional or shadowing instabilities. The assumption of nonlocality has been somehow reinforced by some experiments. Thus, in $\mathrm{Cu}$ electrodeposition studies, ${ }^{17,34}$ it was found that anomalous scaling appeared for conditions in which the ratio of the current density to its diffusion-limited value was relatively high. More specifically, in the notation of the present work, Huo and Schwarzacher ${ }^{17}$ found that $\beta_{\mathrm{loc}}$ and $\alpha_{\mathrm{loc}}$ did not depend on how close or far the system was from the diffusional limit. In contrast, the values of the global exponent did depend on this condition. In fact, anomaly appeared in this system (i.e., $\beta^{*}>0$, note that our $\beta^{*}$ is denoted as $\beta_{\text {loc }}$ in Ref. 17) for larger current densities, i.e., under conditions closer to diffusional limitation. Under these conditions, those sites of the growing interface located at higher positions would receive more material than those further away, inducing faster growth at peaks or protrusions than at valleys. ${ }^{17,29,34}$ Although these findings do not prove, unequivocally, that anomalous scaling is the result of diffusional instability, since overpotential effects cannot be definitively ruled out, ${ }^{17,34}$ they strongly suggest such an interpretation. ${ }^{34}$ Moreover, a similar behavior was found for $\mathrm{Cu}$ electroless deposition, where $\beta^{*}$ resulted smallest for those conditions where growth is likely kinetically limited rather than diffusionally limited. ${ }^{29}$ Additional support for this explanation can be found in the experiments on polymer films grown by vapor deposition. For this system, in which (nonlocal) bulk diffusion was proved to play a key role, anomalous scaling was indeed observed, ${ }^{32}$ in other words, it seems that nonlocality may play a crucial role in anomalous scaling systems.

Alternatively, many studies on film growth by sputtering report high values of $\beta$, typically in the range $0.4-0.8 .^{35-41} \mathrm{In}$ our case, for instance, $\beta$ is equal to 0.67 . This behavior is shared by many of the surface growth systems displaying anomalous scaling. Most of these studies, in which the global and local exponents were analyzed, exhibited $\beta$ values larger than 0.5. Again, this scenario somehow resembles the values of $\beta$ in some experiments on electrodeposition where anomalous scaling has been reported. ${ }^{17,28,29}$ In principle, this behavior (i.e., high $\beta$ values) suggests the existence of a destabilizing growth mechanism. In our sputtering system, Ti species are sputtered from the target and subsequently deposited on the substrate, whose temperature ranges from room temperature, at the first stage of the deposition, to temperatures around $65-70{ }^{\circ} \mathrm{C}$ when the deposition process ends. Besides, the $\mathrm{N}$ active species present in the plasma impinge on the growing film with a sticking coefficient close to $1 .{ }^{42}$ Under our pressure-deposition conditions, the system is below the thermalization threshold ${ }^{43}$ and the average mean free path of the Ti species $\lambda$ is $\approx 5 \mathrm{~cm}$, which is of the order of the target-substrate distance. This implies that $\mathrm{Ti}$ and $\mathrm{N}$ species do not react within the plasma, but rather, they do so at the growing film surface. Thus, we can assume, to a first approximation, that the impinging particles are $\mathrm{Ti}$ and $\mathrm{N}$ species following ballistic trajectories. Furthermore, it is known that the flux of sputtered target particles follows a cosine distribution in sputtering processes. ${ }^{44}$ These facts indicate that shadowing effects could play an important role in the film dynamics as other authors have already suggested. ${ }^{16,36,41}$ Shadowing effects also imply that regions located at higher surface positions (peaks or protrusions) grow faster at the expense of others (valleys), which is known to lead to unstable growth morphologies with large $\beta$ values, ${ }^{45}$ analogous to the diffusional instabilities found in electrodeposition. ${ }^{17,29}$

Available models of shadowing in $2+1$ dimensions predict $1 / z=1 / 3$ and $\beta=1 .{ }^{46}$ Note that, whereas the coarsening exponent in our system is relatively close to the predicted value, the experimental value of $\beta$ is clearly smaller. However, recently, Yu and Amar have studied theoretically the dynamic scaling of ballistic deposition with shadowing on unidimensional substrates. ${ }^{44}$ In this work, they consider ex- 
plicitly two conditions for the angular distribution of the impinging particles: uniform angular distribution and cosine distribution. They found that the former condition, which is the standard one used in the models of shadowing, corresponds to an anisotropic flux of particles on the growing surface. In contrast, the latter one, which is close to that taking place in sputter deposition, implies an isotropic flux. Moreover, they reported that, for a cosine distribution, the $\beta$ value was smaller than those obtained for the anisotropic flux that was close to 1 . Thus, shadowing effects could lead to still high $\beta$ values for sputtering deposition, but clearly smaller than the value $\beta \approx 1$ predicted by the previous models. It should be stressed that for our system conditions, where $\lambda$ is close to the target-substrate distance, the impinging particles follow ballistic trajectories as in the model of Yu and Amar. Moreover, in their model they found that the evolving interface displayed both anomalous scaling and multiscaling ${ }^{44}$ as in our case. Unfortunately, this study was performed only for unidimensional substrates, whereas realistic substrates are two-dimensional, which prevents a direct comparison of the experimental exponents to the theoretical ones. ${ }^{1,3}$ However, the trend of smaller $\beta$ values for a cosine distribution should be obeyed also on two-dimensional substrates. Moreover, the fact that many of the reported $\beta$ values for sputtering systems lie in the 0.4-0.6 range supports this interpretation.

With this overall picture in mind, we can conclude that the anomalous scaling behavior found for TiN films grown by sputtering on $\mathrm{Si}(100)$ substrates, is related to some nonlocal effect taking place during the growth process. Moreover, we argue that it is probably due to shadowing effects operating during the deposition process under our experimental conditions. Besides, we want to stress that the fact that both multiscaling and anomalous scaling are present in our system may drive some theoretical efforts to generalize $1+1$ theories and models to $2+1$ systems.

\section{ACKNOWLEDGMENTS}

We wish to thank E. Moro for useful discussions. Financial support is acknowledged from Spanish MCyT Grants No. MAT 2002-04037-C03-03 and No. BFM 2003-07749C05-01, -02, and -05; Comunidad Autónoma de Madrid, Grant No. GR/MAT/0431/2004, European Community Grant No. G5RD-CT-2000-00333, Centre of Excellence CE PI SAS, Contract No. I/2/2005, and Slovak Grant Agency for Science VEGA, Grant No. 2/6030/26.
*Corresponding author. Electronic adress: 1vb@icmm.csic.es

${ }^{1}$ A. L. Barabási and H. E. Stanley, Fractal Concepts in Surface Growth (Cambridge University Press, Cambridge, England, 1995).

${ }^{2}$ P. Meakin, Fractals, Scaling and Growth far from Equilibrium (Cambridge University Press, Cambridge, England, 1998).

${ }^{3}$ J. Krim and G. Palasantzas, Int. J. Mod. Phys. B 9, 599 (1995).

${ }^{4}$ R. Cuerno and L. Vázquez, in Advances in Condensed Matter and Statistical Physics, edited by E. Korutcheva and R. Cuerno (Nova Science Publishers, New York, 2004).

${ }^{5}$ M. Stoiber, M. Panzenböck, C. Mitterer, and C. Lugmair, Surf. Coat. Technol. 142-144, 117 (2001).

${ }^{6}$ W. H. Lee, Y. L. Kuo, H. J. Huang, and C. P. Lee, Mater. Chem. Phys. 85, 444 (2004).

${ }^{7}$ G. S. Chae, H. S. Soh, W. H. Lee, and J. G. Lee, J. Appl. Phys. 90, 411 (2001).

${ }^{8}$ A. Katz, A. Feingold, S. J. Pearton, S. Nakahara, M. Ellington, U. K. Chakrabarti, M. Geva, and E. Lane, J. Appl. Phys. 70, 3666 (1991).

${ }^{9}$ K. C. Park, K. B. Kim, I. J. M. M. Raaijmakers, and K. Ngan, J. Appl. Phys. 80, 5674 (1996).

${ }^{10}$ F. Ojeda, R. Cuerno, R. Salvarezza, and L. Vázquez, Phys. Rev. Lett. 84, 3125 (2000); F. Ojeda, R. Cuerno, R. Salvarezza, F. Agulló-Rueda, and L. Vázquez, Phys. Rev. B 67, 245416 (2003).

${ }^{11}$ J. Santamaría, M. E. Gómez, J. L. Vicent, K. M. Krishnan, and I. K. Schuller, Phys. Rev. Lett. 89, 190601 (2002).

${ }^{12}$ J. Soriano, J. J. Ramasco, M. A. Rodríguez, A. HernándezMachado, and J. Ortín, Phys. Rev. Lett. 89, 026102 (2002).

${ }^{13}$ J. M. López, M. A. Rodríguez, and R. Cuerno, Physica A 246, 329 (1997).
${ }^{14}$ J. J. Ramasco, J. M. López, and M. A. Rodríguez, Phys. Rev. Lett. 84, 2199 (2000).

${ }^{15}$ J. M. López, M. A. Rodríguez, and R. Cuerno, Phys. Rev. E 56, 3993 (1997).

${ }^{16}$ J. H. Jeffries, J. K. Zuo, and M. M. Craig, Phys. Rev. Lett. 76, 4931 (1996); S. Morel, J. Schmittbuhl, J. M. López, and G. Valentin, Phys. Rev. E 58, 6999 (1998).

${ }^{17}$ S. Huo and W. Schwarzacher, Phys. Rev. Lett. 86, 256 (2001).

${ }^{18}$ J. Asikainen, S. Majaniemi, M. Dubé, and T. Ala-Nissila, Phys. Rev. E 65, 052104 (2002).

${ }^{19}$ J. Asikainen, S. Majaniemi, M. Dubé, J. Heinonen, and T. AlaNissila, Eur. Phys. J. B 30, 253 (2002).

${ }^{20}$ J. Soriano, J. Ortín, and A. Hernández-Machado, Phys. Rev. E 67, 056308 (2003).

${ }^{21}$ M. A. Auger, R. Gago, M. Fernández, O. Sánchez, and J. M. Albella, Surf. Coat. Technol. 157, 26 (2002).

${ }^{22}$ D. Tsamouras and G. Palasantzas, Appl. Phys. Lett. 80, 4528 (2002).

${ }^{23}$ M. Siegert, Phys. Rev. E 53, 3209 (1996).

${ }^{24}$ R. Kant, Phys. Rev. E 53, 5749 (1996).

${ }^{25}$ J. Schmittbuhl, J. P. Vilotte, and S. Roux, Phys. Rev. E 51, 131 (1995).

${ }^{26}$ S. Das Sarma, S. V. Ghaisas, and J. M. Kim, Phys. Rev. E 49, 122 (1994).

${ }^{27}$ J. Krug, Phys. Rev. Lett. 72, 2907 (1994)

${ }^{28}$ M. Saitou, Phys. Rev. B 66, 073416 (2002); M. Saitou, K. Hamaguchi, and W. Oshikawa, J. Electrochem. Soc. 150, C99 (2003).

${ }^{29}$ N. M. Hasan, J. J. Mallett, S. G. dos Santos Filho, A. A. Pasa, and W. Schwarzacher, Phys. Rev. B 67, 081401(R) (2003).

${ }^{30}$ G. Palasantzas, Phys. Rev. E 56, 1254 (1997). 
${ }^{31}$ L. Vázquez, J. M. Albella, R. C. Salvarezza, A. J. Arvia, R. A. Levy, and D. Perese, Appl. Phys. Lett. 68, 1285 (1996).

${ }^{32}$ Y. P. Zhao, J. B. Fortin, G. Bonvallet, G. C. Wang, and T. M. Lu, Phys. Rev. Lett. 85, 3229 (2000).

${ }^{33}$ J. M. López, M. Castro, and R. Gallego, Phys. Rev. Lett. 94, 166103 (2005).

${ }^{34}$ W. Schwarzacher, J. Phys.: Condens. Matter 16, R859 (2004).

${ }^{35}$ H. You, R. P. Chiarello, H. K. Kim, and K. G. Vandervoort, Phys. Rev. Lett. 70, 2900 (1993).

${ }^{36}$ M. Saitou, A. Makabe, and T. Tomoyose, Europhys. Lett. 52, 185 (2000).

${ }^{37}$ R. M. Öksüzoglu, A. Elmali, T. E. Weirich, H. Fuess, and H. Hahn, J. Phys.: Condens. Matter 12, 9237 (2000).

${ }^{38}$ N. E. Lee, D. G. Cahill, and J. E. Greene, Phys. Rev. B 53, 7876 (1996).
${ }^{39}$ J. Wang, G. Li, P. Yang, M. Q. Cui, X. M. Jiang, B. Dong, and H. Liu, Europhys. Lett. 42, 283 (1998).

${ }^{40}$ J. P. Schlomka, M. Tolan, and W. Press, Appl. Phys. Lett. 76, 2005 (2000).

${ }^{41}$ T. Karabacak, Y. P. Zhao, G. C. Wang, and T. M. Lu, Phys. Rev. B 64, 085323 (2001).

${ }^{42}$ D. L. Smith, A. S. Alimonda, and F. J. von Preissig, J. Vac. Sci. Technol. A 8, 551 (1990).

${ }^{43}$ T. Salditt, D. Lott, T. H. Metzger, J. Peisl, G. Vignaud, P. Høghøj, O. Schärpf, P. Hinze, and R. Lauer, Phys. Rev. B 54, 5860 (1996).

${ }^{44}$ J. Yu and J. G. Amar, Phys. Rev. E 66, 021603 (2002).

${ }^{45}$ D. Le Bellac, G. A. Niklasson, and C. G. Granqvist, Europhys. Lett. 32, 155 (1995).

${ }^{46}$ J. H. Yao and H. Guo, Phys. Rev. E 47, 1007 (1993). 